\title{
111
}

\section{Object-oriented Specification and Design of User-interfaces}

\author{
Andrew Hussey \\ Software Verification Research Centre \\ School of Information Technology \\ The University of Queensland \\ Brisbane, Qld, 4072, Australia \\ email: ahussey@cs.uq.edu.au
}

\begin{abstract}
The specifier of a user-interface describes user-perceivable functions and information organisation in an implementation-independent way. The designer is concerned with both the presentation and software structure of the user-interface. The path from specification to software design can be assisted by design patterns. In this paper I examine techniques for specifying and designing both the presentation and software aspects of user-interfaces. I outline my research strategy for creating a specification and design framework for user-interface development.
\end{abstract}

KEYWORDS Human-computer interface, specification, design, object-orientation, formal methods.

\section{Introduction}

The user-interface of a system is the view of the system that is provided to users. So, the development of a user-interface is a specialised software engineering activity. Just as in other branches of software engineering, there is a need to describe user-interfaces both abstractly (specification) and at a more concrete implementation-dependent level (design). Formal methods have shown promise as a technique for specification and design in other branches of software engineering. In this thesis I address the question as to how they may be applied to user-interface specification and design.

I have conducted exploratory research projects on both specification and software design of userinterfaces. These exploratory projects have resulted in the selection of Object- $Z$ as a formal specification and design language. Also as a result of my work on these projects, I have generated two specific aims:
- generate a specification and design framework based on the Object- $Z$ language aimed at assisting the development of user-interfaces;

- produce guidelines (i.e., patterns) for designing the user-interface components of interactive systems, and the components of the functional core that directly communicate with the userinterface.

In Section 2, I describe my research plan to meet these objectives. Section 3 considers my view of userinterface specification, developed as a result of my research. Section 4 deals with my research results on design of user-interfaces and Section 5 describes my future work. 


\section{Research Plan}

I have produced specifications and designs for a variety of case studies. By taking a case-study approach, I hope to derive principles of broad applicability to user-interface specification and design. In addition, using case-studies has provided an opportunity to evaluate the strengths and weaknesses of Object- $Z$ as a tool for interactive system modelling. To assess the suitability of the framework, I intend to conduct an experiment in which subjects are requested to construct an interactive system from either an informal or a formal Object- $Z$ design description. Section 5 describes the experiment in more detail.

\section{Specification}

A specification describes the functions that a system provides to its users without detailing how those functions are implemented. Specification is the process of abstractly defining an entity from a certain perspective. The user-interface of a system can be defined as the view of a system that is provided to users. Therefore a user-interface specification is an abstract description of a system from the perspective of its users; such a specification is useful for developing user-interface designs.

A specification of an interactive system's userinterface indicates which functional aspects of the system (state and operations) are perceivable by the user and the logical organisation of information provided by the interface. Such a specification does not describe the presentation (e.g., visual/audio) aspects of the user-interface. The presentation aspects are design concerns (i.e., how the user-interface is implemented, rather than an abstract description of what is to be implemented). For this reason, a specification of a user-interface provides only part of the information needed to construct an implementation.

User-interfaces can be specified as a collection of interacting agents using an agent-based language (Abowd, 1991). Agents have state, operations upon that state and may respond to and initiate events (Abowd, 1991). In an interactive system, agents are presented to users. The agent and its presentation together define an interactor (Coutaz et al., 1995). An interactor has a presentation aspect which reflects the internal state of the

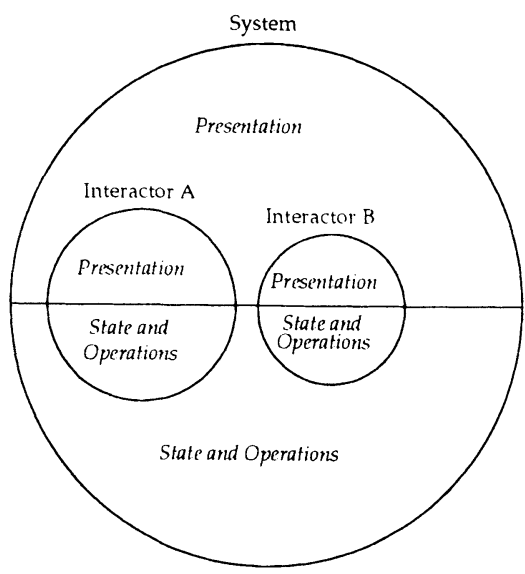

Figure 1: An interactor design

application (Duke and Harrison, 1993) (i.e., as defined by the corresponding agent). Interactors can be combined to form larger (more complex) interactors. Each agent is a specification (i.e., state and operations) of a corresponding interactor (i.e., agent + presentation). The interactor specifications define the logical organisation of information presented by the user-interface and therefore provide a starting point for design of the presentation.

Object-Z (Duke et al., 1991), an object-oriented extension of $Z$ (Spivey, 1992), can be used as a notation for interactor-based specification.

\section{Designing User-Interfaces}

The design of the presentation of a user-interface is distinct from and precedes the software design of the user-interface. Such a design defines how inputs and outputs of the system are presented. Presentation designs may be structured using interactors. An interactor framework may be constructed, using Object-Z, to support presentation design. The design case studies have generated an interactor-based "widget library" for describing the presentation of a user-interface in terms of common "widgets". A widget is a conceptually distinct sub-component of the presentation of a system. Widgets have characteristic appearance and behaviour. (Common widgets 
include buttons and scrollbars. Figure 1 shows a presentation design that consists of several interactors. A widget-based design identifies the correspondence between interactors in a design and widgets in a widget library.

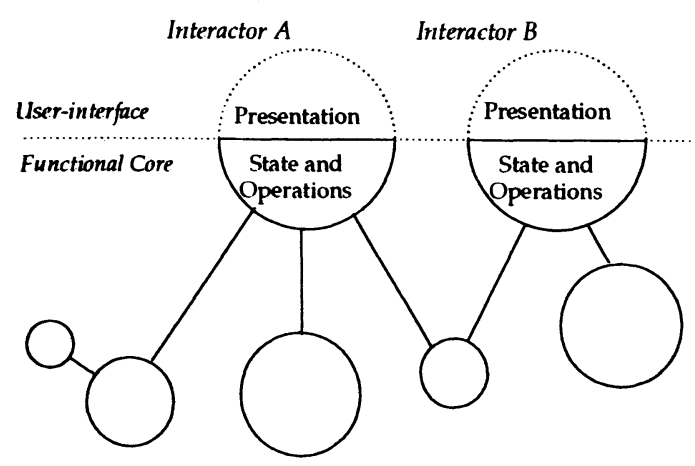

Figure 2: Interactor-based architecture

Software design requires the selection of an architecture which defines the system components and the communication between components. In an interactor-based architecture, the system is divided into interactors. Each interactor consists of a functional component and an interface component. The functional components are "sockets" which provide access to a functional-core. The functional core defines that part of an interactive system which is "invariant to change in the media used for interacting with the user" (Conference Working Group, 1990). Interactor specifications define the functional subcomponent of one or more design interactors and their interface to the presentation sub-component (see Figure 2). The specification defines a view of the functional-core - it does not necessarily define the entire functional-core of the system.

The path from specification to design can be assisted by design patterns. Patterns divide solutions into their elementary parts which can be later recombined and reused, providing a way of describing designs that communicates the reasons for design decisions (Gamma et al., 1994). Patterns can help designers recognise the appropriate software components required to implement each specification interactor. Such patterns can guide the designer in selecting both the appearance of the system and an architectural design suited to the requirements.

\section{Future Work}

In my future work I intend to analyse the extent to which my techniques are applicable to all the case studies and the extent to which they may be broadly applicable to all interactive systems. The results of this cross-study analysis may require some modification of the modelling techniques and design patterns.

I also intend to conduct an experimental study in which subjects construct a user-interface from a userinterface design. Subjects will be provided with either an informal presentation design (the "control" group) or a formal Object- $Z$ presentation design and will be asked to construct the user-interface. Both experimental groups will use the same programming language; likewise, both designs will describe the same user-interface. Subjects will receive instruction in reading Object- $Z$ designs and user-interface programming in a workshop format prior to the experiment. Subjects will be requested to note their activities during the course of constructing the userinterface. Subjects will be able to consult a tutor during the experiment and questions they ask will be noted.

My experiment will enable an assessment of the utility of my specification and design techniques. Assessment will be against effort expended by each group, questions asked and conformance to the intended design. Again, the results of the experiment may lead to modification of the modelling techniques and design patterns.

\section{Conclusions}

My thesis aims to find formal techniques for the specification and design of user-interfaces. The results of my research to date are summarised as follows. A user-interface specification indicates which functional aspects of a system are perceivable by the user and the logical organisation of information provided by the interface. User-interface specifications provide a basis for design of the presentation aspects of the user-interface. It provides a formalisation of the anticipated user model. A design defines the presentation and software structure of a system. Both 
specifications and designs can be structured using interactors. Patterns assist interactor-based software design.

My work makes several valuable contributions:

- formalising specification and design of userinterfaces provides developers with many of the benefits of formal methods (e.g., clarity, precision and conciseness).

- patterns for the transformation process from specification to software design, help streamline the development process, allowing more attention to be given to user-interface design.

- a specification and design framework helps developers ensure that the user-interfaces they develop meet the user's functional requirements.

\section{References}

Abowd, G. D. (1991). Formal descriptions of user interfaces. Colloquium Digest, 192:7/1-7/3.

Conference Working Giroup (1990). Concepts, Methods, Methodologies Working Group. In Duce, D. A., Gomes, M. R., Hopgood, F. R. A., and Lee, J. R., editors, User Interface Management and Design: Proceedings of the Workshop on User Interface Management Systems and Environments, pages 35-49. Springer-Verlag.

Coutaz, J., Nigay, L., and Salber, D. (1995). AgentBased Architecture Modelling for Interactive Systems. Technical Report SM/WP53, LGI-IMAG, (irenoble. ESPRIT BRA 7040 Amodeus-2.

Duke, D. J. and Harrison, M. D. (1993). Abstract Interaction Objects. Computer Graphics Forum Conference Issue, 12(3):25-36.

Duke, R., King, P., Rose, G., and Smith, G. (1991). The Object-Z Specification Language Version 1. Software Verification Research Centre TR91-1, The University of Queensland.

Gamma, E., Helm, R., Johnson, R., and Vlissides, J. (1994). Design Patterns: Elements of Reusable Object-Oriented Software. Addison-Wesley.
Spivey, J. M. (1992). The $Z$ notation: a reference manual. Prentice-Hall, 2nd edition. 